\title{
Fluctuating Motion in an Active Environment
}

\author{
Christian Maes (1) \\ Instituut voor Theoretische Fysica, KU Leuven 3001, Belgium
}

(Received 28 May 2020; accepted 17 September 2020; published 9 November 2020)

\begin{abstract}
We derive the fluctuation dynamics of a probe in weak coupling with a living medium, modeled as particles undergoing an active Ornstein-Uhlenbeck dynamics. Nondissipative corrections to the fluctuation-dissipation relation are written out explicitly in terms of time correlations in the active medium. A first term changes the inertial mass of the probe as a consequence of the persistence of the active medium. A second correction modifies the friction kernel. The resulting generalized Langevin equation benchmarks the motion induced on probes immersed in active versus passive media. The derivation uses nonequilibrium response theory.
\end{abstract}

DOI: 10.1103/PhysRevLett.125.208001

Introduction.-For more than a century, the paradigm of Brownian motion and its variations have been used in mesoscopic physics to describe fluctuating dynamics. Their impact has even been broader as they have advanced the use of stochastic processes and the study of dynamical fluctuations in physical phenomena and far beyond. The central idea is atomism, that is, that the surrounding heat bath consists of many fast moving atoms or molecules colliding with the particle, simultaneously adding friction and noise. Under the systematics of the van Hove or weak coupling limit or as an application of the Zwanzig-Mori formalism, a Markov process is obtained for the particle motion suspended in that equilibrium environment [1-3]. The resulting diffusion satisfies the fluctuation-dissipation relation (FDR) as a result of time-reversal invariance of the underlying Hamiltonian dynamics for system and environment together. It implies that the induced motion satisfies detailed balance.

A similar strategy can be applied when particles are in contact with spatially well-separated equilibrium reservoirs, whether mechanical, chemical, or thermal. Now and again in the suitable scaling limit, local detailed balance governs the fluctuating dynamics of the system [4-7]. It allows a physically reasonable ground to study transport properties and fluctuations in the created currents. The revival of nonequilibrium statistical mechanics during the last two decades has mostly concerned such systems.

This Letter tackles a third stage of that problem in which we consider a probe (or tracer) coupled directly to a nonequilibrium medium. In many interesting cases that medium is active, where particle locomotion is coupled with internal or external nonequilibrium degrees of freedom. Typical examples include self-propelled bacteria or small beads, Janus particles, that can be optically or mechanically activated to run and tumble [8-10]. It is often an excess in dynamical activity, a nonphoretic driving or, more generally, a breaking of the Einstein relation that results in such nonequilibrium behavior. Various simplified models have been proposed for such media, and this Letter takes the active Ornstein-Uhlenbeck (AOU) process for the dynamics of their particles. The main specific question is how to integrate out the AOU particles coupled to a passive probe and thus derive the induced motion of the probe. Interestingly, we will discover mass generation on the probe from the persistence in the medium and additional (possibly negative) friction breaking the Einstein relation.

The interest in benchmarking fluctuating motion in active environments is clear from the wealth of studies in experimental and theoretical microrheology and biophysics, witnessed, e.g., by [11-18]. For example, the influence of active or critically driven droplets on, e.g., transcriptional regulation has recently moved to the foreground $[19,20]$. The general question is how nonequilibrium features in the active particles' motion are transferred to the probe dynamics. Alternatively, probe motion can provide information about the condition of the active medium, and its analysis may lead to diagnosing the quality or efficiency of life processes. But we first need to understand accurately how activity parameters such as the persistence play a role in the resulting fluctuating dynamics. Such studies are also supported by the pleasant fact that the possibilities for observing and manipulating mesoscopic kinetics have been growing sensationally thanks to, e.g., optical tweezing and fast-camera tracking. Developments using a trajectory-based approach to response are in line with these new tools. Theoretical progress on the nature of the induced motion has been reported in [12,21-26], using a variety of approximations and/or numerical work to characterize interactions and induced forces. To the best of our knowledge, however, the fluctuating dynamics of one or more probes in an active bath has not been derived. We desire a constructive approach enabling us to see in exactly what sense the probe gets activated. The methodology for such studies has 
been developed first in [27,28] for deriving the motion in nonequilibrium media satisfying local detailed balance. Yet, in the present case, the translational motion of the active particles making the medium is non-Markovian and far from even local detailed balance.

Active Ornstein-Uhlenbeck medium.-A commonly used model for an active overdamped dynamics via introducing of an Ornstein-Uhlenbeck noise (see [16,29-37]). The simplicity of that choice allows a rich variety of simulation and theoretical studies, including of confinement and interactions. That model has been studied in the context of motility-induced phase transitions [30,38] but also in the context of modifications in glass physics [39] or other phenomena such as pressure on walls [40]. The AOU dynamics is written in Eqs. (2) and (3) for coupling with the probe position. The activity resides in the colored noise $v(s)$ reflecting the pushes from many hidden active components such as molecular motors. In that sense, an AOU process is thought to model the dynamics of tracers in living systems [41-43]. Here we use a probe to trace a medium of AOU particles.

We refer to a probe here instead of a particle because the constituents of the active medium are named particles. While those active particles usually never represent atoms, ions, or molecules, they are (at least) micron-sized and in contact with an equilibrium environment of thermal or chemical baths for dissipating their waste energy. The probe is even bigger than the active particles and could be a wall with fluctuations visible in the dynamics for probes to tens of a micrometer in size [11]. Nevertheless, our study remains conceptually a direct extension of the derivation of Brownian motion for a colloid suspended in a thermal equilibrium bath. The same assumptions as for deriving Brownian motion are made concerning timescale separation and weak coupling except for the equilibrium condition of the bath. Even local detailed balance is indeed violated in the active medium. We will not use knowledge of the stationary distribution of the medium, even though many aspects are known for the simplest versions $[37,44,45]$. Instead, we use a trajectory-based response theory [46] that is both applicable to more complicated situations and useful for obtaining the resulting fluctuating dynamics in explicit measurable quantities.

Setup around quasistatic limit.-After denoting $Y_{t}$ as the position of the probe with mass $M$ at time $t$, its Newton equation (in one-dimensional notation always for simplicity) reads

$$
M \ddot{Y}_{t}=-\lambda \sum_{i=1}^{N}\left[Y_{t}-x_{i}(t)\right]
$$

and couples with $N$ AOU particles having positions $x_{i}(t)$. The linearity of the coupling is not to be taken as physically literal but only as a mathematical simplification. For an interaction potential $\Phi\left[\left|Y_{t}-x_{i}(t)\right|\right]$ with short-range $\delta$, we should think of $\lambda=\rho \delta \Phi^{\prime \prime}(0)$ (positive or negative) with $\rho$ the medium density, as measuring the effective spring constant.

The AOU overdamped dynamics is (for each particle)

$$
\begin{gathered}
\dot{x}(s)=\mathcal{E} v(s)+\lambda \mu\left[Y_{s}-x(s)\right]-\mu V^{\prime}[x(s)], \\
\tau \dot{v}(s)=-v(s)+\sqrt{2 R} \xi(s) .
\end{gathered}
$$

Their activity depends on a persistence time $\tau$ and noise amplitudes $\mathcal{E}$ and $R$. The $\xi(s)$ is standard white noise representing further hidden degrees of freedom. For $\tau=0$, $v(s)=\sqrt{2 R} \xi(s)$ can be substituted in Eq. (2) and the medium becomes passive, characterized by an overdamped Langevin dynamics in contact with an equilibrium bath at temperature $T=\mathcal{E}^{2} R /\left(k_{B} \mu\right)$ for mobility $\mu$. We use the same notation in the active case as well, even though $T$ no longer represents the physical temperature then. The potential $V=V_{N}$ on each AOU particle may effectively represent a mean-field interaction and may depend on the density profile as well, which gives interesting possibilities for, e.g., motility-induced phase transitions [38]. To make sure, other forces and noise can be added to the Newton Eq. (1), even to the extent of making the motion overdamped. All those would be additive to Eq. (1). We focus therefore on the main subject, which instead is to integrate out the active medium from Eq. (1).

We proceed under the usual assumptions where the probe's motion is derived as an expansion around the quasistatic limit, also known as the Born-Oppenheimer approximation or adiabatic limit (see, e.g., [1-3]). That limit means that the probe is much slower with respect to the AOU particles, enabling a study in the spirit of EinsteinLaub theory for probes in electromagnetic media. Second, we assume a weak coupling $\lambda$, possibly scaling with $N$ and the duration of the coupling, to ensure compatibility with the quasistatic regime.

As quasistatic reference process, we take the dynamics

$\dot{x}(s)=\mathcal{E} v(s)+\lambda \mu\left[Y_{t}-x(s)\right]-\mu V^{\prime}[x(s)], \quad s \leq t$,

which is Eq. (2) but for held-fixed probe position $Y_{t}$. The Ornstein-Uhlenbeck noise $v(s)$ still works from Eq. (3). With Eq. (4), the resulting $x(s)$ process enjoys a nonequilibrium non-Markovian steady condition for which we denote the statistical averaging by $\langle\cdot\rangle_{\tau}^{\lambda}$ —of course also depending on the instantaneously static probe position $Y_{t}$ except for $\lambda=0$. Informally, the $s$ time of the AOU particles in Eq. (4) runs much faster than the $t$ time of the probe. In that quasistatic approximation, Eq. (1) becomes

$$
M \ddot{Y}_{t}=-\lambda N\left(Y_{t}-\langle x\rangle_{\tau}^{\lambda}\right)
$$

as if the active particle positions have relaxed to their stationary expectation for the instantaneous probe position $Y_{t}$. 
Result.-The main result of this Letter can be summarized in the following structure for the induced probe's motion. Suppose the original mass of the probe is rescaled as $M=\lambda_{0} N m$ with $m$ a reference mass and $\lambda=g \lambda_{0}$ with $\lambda_{0}$ dimensionless. Integrating out the AOU medium from Eq. (1), we find

$$
\left(m+g \tau^{2} \frac{\lambda \mu}{2 a}\right) \ddot{Y}_{t}=-g\left(Y_{t}-\langle x\rangle_{\tau}^{\lambda}\right)-\gamma \dot{Y}_{t}+\sqrt{2 \Gamma} \xi_{t},
$$

with rescaled mass shifted proportional to $\tau^{2}$ (persistence) and the coupling $a^{-1}=\int_{0}^{\infty} \mathrm{d} s\langle x(s) ; v(0)\rangle_{\tau}^{0} /(\mathcal{E} R)$ between spatial displacement and the Ornstein-Uhlenbeck noise in the medium. The friction in Eq. (6)

$$
\gamma=\frac{\Gamma}{2 T}+\frac{g \lambda \mu}{2 T} \int_{0}^{\infty} \mathrm{d} s s\left\langle x(s) ; V^{\prime}[x(0)]\right\rangle_{\tau}^{0}
$$

connects its first term with the white noise amplitude $\Gamma=g \lambda \int_{0}^{\infty} \mathrm{d} s\langle x(s) ; x(0)\rangle_{\tau}^{0}$. The second term carries no definite sign and depends on the correlation between the effective force on an AOU particle and its displacement. The mass shift and the extra contribution to the friction are the main changes due to the activity.

In Eq. (6), we took a Markov approximation of the following more accurate description of the induced probe motion:

$$
\begin{gathered}
M \ddot{Y}_{t}=-\lambda N Y_{t}+\lambda N\langle x\rangle_{\tau}^{\lambda} \\
-\frac{1}{2} \lambda^{2} N \int_{0}^{\infty} \mathrm{d} s K(s) \dot{Y}_{t-s}+\sqrt{\lambda^{2} N} \eta_{t} \\
-\frac{1}{2} \lambda^{2} N \int_{0}^{\infty} \mathrm{d} s \mathcal{D}(s) \frac{Y_{t}-Y_{t-s}}{s} \\
-\tau^{2} \mathcal{E} \frac{\lambda^{2} N}{2 T} \int_{0}^{\infty} \mathrm{d} s \ddot{Y}_{t-s}\langle x(s) ; v(0)\rangle_{\tau}^{0} .
\end{gathered}
$$

We ignore terms of order $O\left(\lambda^{3} N\right)$. All expectations $\langle\cdot\rangle_{\tau}^{\lambda}$ refer to averages over the stationary AOU medium defined from Eq. (4). The first line in Eq. (8) reflects the quasistatic limit, Eq. (5), of leading order for small $\lambda$.

In Eq. (9), the friction kernel

$$
K(s)=\frac{1}{T}\langle x(s) ; x(0)\rangle_{\tau}^{0}=\frac{1}{T}\left\langle\eta_{s} ; \eta_{0}\right\rangle_{\tau}^{0}
$$

is proportional to the noise covariance as in the standard FDR. The noise $\eta_{t}$ is stationary, of mean zero, and picks up the distribution of

$$
\eta_{s}=\frac{1}{\sqrt{N}} \sum_{i=1}^{N}\left[x_{i}(s)-\langle x\rangle_{\tau}^{\lambda}\right]
$$

in the AOU medium following Eq. (1). The terms in Eqs. (8) and (9) would also be present for a passive medium $\tau=0$; below we explain the factor $1 / 2$.

The third line in Eq. (10) is an additional friction, with kernel

$$
\mathcal{D}(s)=\frac{\mu}{T} s\left\langle x(s) ; V^{\prime}[x(0)]\right\rangle_{\tau}^{0},
$$

which is unbalanced by the noise. Note that $\left(Y_{t}-Y_{t-s}\right) / s \simeq$ $\dot{Y}_{t-s}$ for small $s$, which is most relevant as $\mathcal{D}(s)$, is expected to decay exponentially fast [see Eq. (17)]. As we show in Eq. S9 of the Supplemental Material [47], in the passive case $\tau=0$

$$
\int_{0}^{\infty} \mathrm{d} s \mathcal{D}(s) \frac{Y_{t}-Y_{t-s}}{s}=\int_{0}^{\infty} \mathrm{d} s K(s) \dot{Y}_{t-s},
$$

which then removes the factor $1 / 2$ from Eq. (9) to install the standard FDR from Eq. (12). In the active case, however, Eq. (10) is the explicit source of violation of the FDR in the induced fluctuating dynamics. We call Eq. (14) the nondissipative friction kernel as Eq. (10) corresponds to the socalled frenetic contribution in linear response of the same order as the dissipative friction with the kernel, Eq. (12) (see [46,52]). The kernel, Eq. (14), may produce a negative contribution to the friction. Indeed, its sign is decided from the time correlation between AOU position $x(s)$ and the effective force $-V^{\prime}[x(0)]$. Imagine, for example, the (many) $N$ particles, each obeying Eq. (2) but replacing there $V \rightarrow \Phi$ describing interactions

$$
\Phi\left(x_{1}, \ldots, x_{N}\right)=\sum_{i, j=1}^{N} W_{N}\left(x_{i}, x_{j}\right)+\sum_{i=1}^{N} \phi\left(x_{i}\right) .
$$

For such interacting dynamics, the AOU particles may show a motility-induced phase separation $[29,38]$. A meanfield version is readily obtained when $W_{N} \propto W / N$ with $W(x, y)=W(x)+m_{1} W^{\prime}(x)+m_{2} W^{\prime \prime}(x)$ independent of $y$ and with $m_{i}$ describing effectively the density and its spreading. A typical choice in Eq. (2) would then be to take

$V^{\prime}(x)=\phi^{\prime}(x)+W^{\prime}(x)+m_{1} W^{\prime \prime}(x)+m_{2} W^{\prime \prime \prime}(x)$,

making an AOU process, Eq. (2), where the interaction and density are parameterized via the parameters $m_{1}, m_{2}$. The different terms in Eq. (16) may naturally produce different signs in Eq. (14). In particular, it is allowed to have a contribution $k\langle x(s) ; x(0)\rangle_{\tau}^{0}<0$ for $k<0$ in Eq. (14) without breaking the stability of the AOU process. It is well known that, for active particles, trapping may lead to clustering at the boundaries of the trap, which is an effective repulsion from the center of the trap [53]. Heuristically, it should also not be too surprising to imagine that persistent flocking carries the probe, transporting 
energy from the active medium to the probe. Negative differential mobility has been found before due to frenetic causes in, e.g., [54,55].

The final line in Eq. (11) is explicit in the persistence $\tau$ and introduces extra inertia to the probe. The bare mass $M$ of the probe gets modified via the time correlation of the position $x(t)$ and the free Ornstein-Uhlenbeck noise $\mathcal{E} v(s)=\dot{x}(s)+\mu V^{\prime}[x(s)], \quad$ as summarized below in Eq. (19). That can be seen as an instance of entropy production (due to the active medium) renormalizing the inertial mass of the immersed probe. A similar effect has been observed in the stabilization of a probe when in contact with a driven environment [56].

In order to understand the dependence of the time correlations appearing in Eqs. (8)-(10) on the persistence $\tau$, we have calculated them for the fully linear case where $V(x)=k x^{2} / 2$ for spring constant $k$. Details are presented in Section II of the Supplemental Material [47]. With $a=\mu(k+\lambda)$, the explicit results are

$$
\begin{gathered}
\langle x(s) ; x(0)\rangle_{\tau}^{\lambda}=\left[\tau e^{-s / \tau}-\frac{1}{a} e^{-a s}\right] \frac{R \mathcal{E}^{2}}{a^{2} \tau^{2}-1}, \\
\left\langle x_{s} ; v_{0}\right\rangle_{\tau}^{\lambda}=\frac{R \mathcal{E}}{a \tau-1}\left\{e^{-s / \tau}-e^{-a s} \frac{2}{a \tau+1}\right\} .
\end{gathered}
$$

The correlation Eq. (18) gives the behavior for the kernel in Eq. (11). For large $a \tau \gg 1$ (far-from-equilibrium regime), we have $\left\langle x_{s} ; v_{0}\right\rangle^{0} \rightarrow(R \mathcal{E} / a)(1 / \tau) e^{-s / \tau}$, which, according to Eq. (11), means that the inertial mass of the probe shifts as

$$
M \rightarrow M+\tau^{2} \mathcal{E} \frac{\lambda^{2} N}{2 T} \frac{R \mathcal{E}}{a}=M+\tau^{2} \frac{\lambda^{2} N}{2 k}
$$

from the influence of the (highly persistent) active medium. The explicit result Eq. (19) is for the correlation Eq. (18) in the linear AOU system but the interpretation is wider: the mass shift is proportional to the persistence over the trapping, which is typically a measure of the variance in the position of active particles.

For intermediate regimes, there are two timescales in the kernels determined by $\tau$ and $a^{-1}$. As already mentioned, for small persistence we recover the standard Langevin equation in the limit $\tau \ll a^{-1}$.

Derivation of the fluctuation dynamics.-The slowly moving probe exerts a stimulus to the AOU medium. The first correction around the quasistatic limit, Eqs. (4)-(5), is provided by the linear response of the medium (see [46] for an introduction). The response or backreaction of the AOU particles determines the fluctuating motion of the probe $[27,28,57,58]$. The linear response around AOU is treated as Section I in the Supplemental Material [47]. We compare two AOU ensembles, one where the path $\left(Y_{s}, s \leq t\right)$ of the probe is given, and one where the probe is held fixed at the final $Y_{t}$.
As we treat the AOU particles effectively as independent, it suffices to investigate the coupled dynamics of just one AOU particle with the probe. We compare the two ensembles by introducing an action $\mathcal{A}(\omega)$ as a function of the AOU-particle trajectories $\omega=\left(x_{s}, s \leq t\right)$ and (not indicated) depending on the probe path $\left(Y_{s}\right)^{t}=\left(Y_{s}, s \leq t\right)$ :

$$
\begin{aligned}
& \operatorname{Prob}\left(\omega \mid Y_{s}, s \leq t\right) \\
& \quad=\exp [-\mathcal{A}(\omega)] \operatorname{Prob}\left(\omega \mid Y_{s}=Y_{t}, \quad \text { for all } s \leq t\right) .
\end{aligned}
$$

The left-hand side gives the weight on trajectories where the AOU particle follows Eq. (2), i.e., is conditioned on $\left(Y_{s}\right)^{t}$. The probability in the right-hand side is the reference weight for AOU-particle trajectories supposing the probe has always been at the (final) position $Y_{t}$. The action $\mathcal{A}$ in Eq. (20) is entirely explicit as computed in Section I of the Supplemental Material [47],

$$
\mathcal{A}(\omega)=-\frac{1}{2 \mathcal{E}^{2}} \int \mathrm{d} s \mathcal{K}(s)\left\{\dot{x}(s)+\mu U^{\prime}[x(s)]\right\},
$$

where the potential is $U(x)=V(x)+\lambda\left(Y_{t}-x\right)^{2} / 2$ and the kernel equals

$$
\mathcal{K}(s)=\frac{\lambda \mu}{R}\left(Y_{s}-Y_{t}-\tau^{2} \ddot{Y}_{s}\right),
$$

which is the function of the difference in probe position $Y_{s}-Y_{t}$ at time $s$. The persistence time $\tau$ enters crucially in the kernel (and action) multiplying $\ddot{Y}_{s}$.

The relation Eq. (20) implies $\left\langle x(t) \mid\left(Y_{s}\right)^{t}\right\rangle=\langle x(t)\rangle_{\tau}^{\lambda}-$ $\langle x(t) ; \mathcal{A}\rangle_{\tau}^{\lambda}$ to first order in $Y_{s}-Y_{t}$. With Eq. (21), we thus obtain the average force of each AOU particle (leaving away subscripts) on the probe, given the probe path $\left(Y_{s}, s \leq t\right)$,

$$
\begin{aligned}
& \left\langle x(t) \mid\left(Y_{s}\right)^{t}\right\rangle \\
& \quad=\langle x(t)\rangle_{\tau}^{\lambda}+\frac{1}{2 \mathcal{E}^{2}} \int_{-\infty}^{t} \mathrm{~d} s \mathcal{K}_{s}\left\langle x(t) ;\left\{\dot{x}(s)+\mu U^{\prime}[x(s)]\right\}\right\rangle_{\tau}^{\lambda} .
\end{aligned}
$$

The covariances $\langle\cdot ; \cdot\rangle_{\tau}^{\lambda}$ are as before in the quasistatic process Eq. (4). Taking small $\lambda \mu\left(Y_{s}-Y_{t}\right)$ means that the probe has not wandered away too far in the past $s \leq t$ over a time $(\lambda \mu)^{-1}$, which combines the coupling to the probe with the mobility of the AOU particles. All terms in Eqs. (9)(11) except the noise follow from Eq. (23) by substituting Eq. (22). For example,

$$
\begin{gathered}
\frac{\mu}{R \mathcal{E}^{2}} \int_{-\infty}^{t} \mathrm{~d} s\left(Y_{s}-Y_{t}\right)\left\langle x(t) ;\left\{\dot{x}(s)+\mu V^{\prime}[x(s)]\right\}\right\rangle_{\tau}^{\lambda} \\
=-\int_{0}^{\infty} \mathrm{d} s\left[K(s) \dot{Y}_{t-s}+\mathcal{D}(s) \frac{Y_{t}-Y_{t-s}}{s}\right]
\end{gathered}
$$


gives the friction terms. The active contribution in the kernel Eq. (22) gives

$$
\frac{\lambda \mu \tau^{2}}{2 R \mathcal{E}^{2}} \int_{-\infty}^{t} \mathrm{~d} s \ddot{Y}_{s}\left\langle x(t) ; \dot{x}(s)+\mu U^{\prime}[x(s)]\right\rangle_{\tau}^{\lambda},
$$

where $\dot{x}(s)+\mu U^{\prime}[x(s)]=\mathcal{E} v_{s}$ in the quasistatic process Eq. (4). Equation (25) gives Eq. (11) in the induced probe dynamics. To summarize, Eqs. (24) and (25) give the force on the probe by each active AOU particle in Eq. (1) such as decomposed in Eq. (23) (up to a factor of $\lambda$ ). The corresponding terms in Eqs. (9)-(11) follow from replacing $U \rightarrow V+O(\lambda)$.

Let us finally consider the noise. The force in Eq. (1) by each AOU particle on the probe is fluctuating as

$$
\begin{aligned}
& \lambda x(t)=\lambda\left\langle x(t) \mid\left(Y_{s}\right)^{t}\right\rangle+\zeta_{t}, \\
& \zeta_{t}=\lambda x(t)-\lambda\left\langle x(t) \mid\left(Y_{s}\right)^{t}\right\rangle,
\end{aligned}
$$

where $\zeta_{t}$ is a "noise" that depends on the AOU medium and that has mean zero for every probe trajectory $\left(Y_{s}\right)^{t}$. The noise $\zeta_{t}$ enters (rescaled) as $\eta_{t}$ in Eq. (9). Its mean $\left\langle\zeta_{t}\right\rangle=0$ and its covariance is

$$
\left\langle\zeta_{s} ; \zeta_{s^{\prime}}\right\rangle=\lambda^{2}\left\langle x(s) ; x\left(s^{\prime}\right)\right\rangle_{\tau}^{0}
$$

in leading order

$$
\frac{\mu}{\mathcal{E}^{2} R}\left\langle\zeta_{s} ; \zeta_{s^{\prime}}\right\rangle=\lambda^{2} K\left(s-s^{\prime}\right)
$$

which is the remnant Eq. (12) of the Einstein relation $\left(k_{B}=1\right)$. As true in the passive case, the noise need not be determined by its second moment. Whether it becomes Gaussian or even white depends on the steady AOU medium and on performing the full weak coupling limit. No complications enter as long as the AOU medium allows sufficient decay of spacetime correlations for the central limit theorem to work.

Conclusions and outlook.-Benchmarking the probe dynamics immersed in an active medium is an important step beyond Brownian motion for tagged particles suspended in equilibrium baths. An expansion around the quasistatic limit is possible by an application of linear response theory around nonequilibrium. The frenetic contribution adds to the dissipative part in the response and produces extra terms in the induced probe dynamics. A first term modifies the effective mass of the probe and is proportional to the persistence time. Persistence is inherited by the probe from its interaction with the active medium, which effectively generates extra inertia for the probe. The second term breaks the Einstein relation by a contribution to the friction that is not compensated by the noise. That "extra" friction depends on the mutual forces between the medium particles and their correlation with the probe position as in the modified Sutherland-Einstein relation $[55,59]$ and in the Harada-nasa equality [60].

The derivation in the present Letter is restricted to AOU particles for the medium. That makes it possible for being explicit in the response formula. The derivation strongly suggests, however, that the same properties and modifications are found in the induced probe dynamics for more general active media. We look forward to experimental tests where both the mass renormalization and the extra (possibly negative) friction are observed. Of substantial interest would be the discovery of the influence of the motility-induced phase transition in the medium. In the present Letter, that was modeled effectively via the potential $V$ in Eq. (16).

[1] D. Chandler, Introduction to Modern Statistical Mechanics, 1st ed. (Oxford University Press, New York, 1987).

[2] R. Zwanzig, Nonequilibrium Statistical Mechanics (Oxford University Press, New York, 2001).

[3] L. Van Hove, Correlations in space and time and born approximation scattering in systems of interacting particles, Phys. Rev. 95, 249 (1954).

[4] H. Tasaki, Two theorems that relate discrete stochastic processes to microscopic mechanics, arXiv:0706.1032v1.

[5] B. Derrida, Non-equilibrium steady states: Fluctuations and large deviations of the density and of the current, J. Stat. Mech. (2007) P07023.

[6] S. Katz, J. L. Lebowitz, and H. Spohn, Stationary nonequilibrium states for stochastic lattice gas models of ionic superconductors, J. Stat. Phys. 34, 497 (1984); Phase transitions in stationary nonequilibrium states of model lattice systems, Phys. Rev. B 28, 1655 (1983).

[7] C. Maes and K. Netočný, Time-reversal and entropy, J. Stat. Phys. 110, 269 (2003).

[8] S. Ramaswamy, Active matter, J. Stat. Mech. (2017) 054002.

[9] É. Fodor and M. C. Marchetti, The statistical physics of active matter: From self-catalytic colloids to living cells, Physica (Amsterdam) 504A, 106 (2018).

[10] M. C. Marchetti, J. F. Joanny, S. Ramaswamy, T. B. Liverpool, J. Prost, M. Rao, and R. Aditi Simha, Hydrodynamics of soft active matter, Rev. Mod. Phys. 85, 1143 (2013).

[11] Xiao-Lun $\mathrm{Wu}$ and A. Libchaber, Particle Diffusion in a Quasi-Two-Dimensional Bacterial Bath, Phys. Rev. Lett. 84, 3017 (2000).

[12] C. Maggi, M. Paoluzzi, L. Angelani, and R. Di Leonardo, Memory-less response and violation of the fluctuationdissipation theorem in colloids suspended in an active bath, Sci. Rep. 7, 17588 (2017).

[13] É. Roldán, J. Barral, P. Martin, J. M. R. Parrondo, and F. Jülicher, Arrow of time in active fluctuations, arXiv:1803 $.04743 v 3$.

[14] P. Bohec, F. Gallet, C. Maes, S. Safaverdi, P. Visco, and F. Van Wijland, Probing active forces via a fluctuation-dissipation relation: Application to living cells, Europhys. Lett. 102, 50005 (2013).

[15] D. T. N. Chen, A. W. C. Lau, L. A. Hough, M. F. Islam, M. Goulian, T. C. Lubensky, and A. G. Yodh, Fluctuations and Rheology in Active Bacterial Suspensions, Phys. Rev. Lett. 99, 148302 (2007). 
[16] C. Maggi, M. Paoluzzi, N. Pellicciotta, A. Lepore, L. Angelani, and R. Di Leonardo, Generalized Energy Equipartition in Harmonic Oscillators Driven by Active Baths, Phys. Rev. Lett. 113, 238303 (2014).

[17] L. Angelani, C. Maggi, M. Bernardini, A. Rizzo, and R. Di Leonardo, Effective Interactions between Colloidal Particles Suspended in a Bath of Swimming Cells, Phys. Rev. Lett. 107, 138302 (2011).

[18] N. Koumakis, A. Lepore, C. Maggi, and R. Di Leonardo, Targeted delivery of colloids by swimming bacteria, Nat. Commun. 4, 2588 (2013).

[19] H. Chen, M. Levo, L. Barinov, M. Fujioka, J. B. Jaynes, and T. Gregor, Dynamic interplay between enhancer-promoter topology and gene activity, Nat. Genet. 50, 1296 (2018).

[20] W.-K. Cho, J.-H. Spille, M. Hecht, C. Lee, C. Li, V. Grube, and II. Cissè, Mediator and RNA polymerase II clusters associate in transcription-dependent condensates, Science 361, 412 (2018).

[21] O. Granek, Y. Baek, Y. Kafri, and A. P. Solon, Bodies in an interacting active fluid: Far-field influence of a single body and interaction between two bodies, J. Stat. Mech. (2020) 063211.

[22] Y. Baek, A. P. Solon, X. Xu, N. Nikola, and Y. Kafri, Generic Long-Range Interactions between Passive Bodies in an Active Fluid, Phys. Rev. Lett. 120, 058002 (2018).

[23] D. Ray, C. Reichhardt, and C. J. O. Reichhardt, Casimir effect in active matter systems, Phys. Rev. E 90, 013019 (2014).

[24] Ran Ni, M. A. Cohen Stuart, and P. G. Bolhuis, Tunable Long Range Forces Mediated by Self-Propelled Colloidal Hard Spheres, Phys. Rev. Lett. 114, 018302 (2015).

[25] L. R. Leite, D. Lucena, F. Q. Potiguar, and W. P. Ferreira, Depletion forces on circular and elliptical obstacles induced by active matter, Phys. Rev. E 94, 062602 (2016).

[26] S. Chaki and R. Chakrabarti, Effects of active fluctuations on energetics of a colloidal particle: Superdiffusion, dissipation and entropy production, Physica (Amsterdam) 530, 121574 (2019).

[27] C. Maes, On the second fluctuation-dissipation theorem for nonequilibrium baths, J. Stat. Phys. 154, 705 (2014).

[28] C. Maes and S. Steffenoni, Friction and noise for a probe in a nonequilibrium fluid, Phys. Rev. E 91, 022128 (2015).

[29] É. Fodor, C. Nardini, M. E. Cates, J. Tailleur, P. Visco, and F. van Wijland, How Far from Equilibrium is Active Matter?, Phys. Rev. Lett 117, 038103 (2016).

[30] T. F. F. Farage, P. Krinninger, and J. M. Brader, Effective interactions in active Brownian suspensions, Phys. Rev. E 91, 042310 (2015).

[31] G. Szamel, E. Flenner, and L. Berthier, Glassy dynamics of athermal self-propelled particles: Computer simulations and a nonequilibrium microscopic theory, Phys. Rev. E 91, 062304 (2015).

[32] É. Fodor, C. Nardini, M. E. Cates, J. Tailleur, P. Visco, and F. van Wijland, How Far from Equilibrium Is Active Matter? Phys. Rev. Lett. 117, 038103 (2016).

[33] R. Wittmann, C. Maggi, A. Sharma, A. Scacchi, J. M. Brader, and U.M.B. Marconi, Effective equilibrium states in the colored-noise model for active matter I. Pairwise forces in the Fox and unified colored noise approximations, J. Stat. Mech. (2017) 113207.
[34] R. Wittmann, U. M. B. Marconi, C. Maggi, and J. M. Brader, Effective equilibrium states in the colored-noise model for active matter II. A unified framework for phase equilibria, structure and mechanical properties, J. Stat. Mech. (2017) 113208.

[35] D. Mandal, K. Klymko, and M. R. De Weese, Entropy Production and Fluctuation Theorems for Active Matter, Phys. Rev. Lett. 119, 258001 (2017).

[36] C. Maggi, U. M. B. Marconi, N. Gnan, and R. Di Leonardo, Multidimensional stationary probability distribution for interacting active particles, Sci. Rep. 5, 10742 (2015).

[37] S. Das, G. Gompper, and R. G. Winkler, Confined active Brownian particles: Theoretical description of propulsion-induced accumulation, New J. Phys. 20, 015001 (2018).

[38] M. E. Cates and J. Tailleur, Motility-induced phase separation, Annu. Rev. Condens. Matter Phys. 6, 219 (2015).

[39] G. Szamel, E. Flenner, and L. Berthier, Glassy dynamics of athermal self-propelled particles: Computer simulations and a nonequilibrium microscopic theory, Phys. Rev. E 91, 062304 (2015)

[40] U. M. B. Marconi and C. Maggi, Towards a statistical mechanical theory of active fluids, Soft Matter 11, 8768 (2015).

[41] E. Ben-Isaac, Y. K. Park, G. Popescu, F. L. H. Brown, N. S. Gov, and Y. Shokef, Effective Temperature of RedBlood-Cell Membrane Fluctuations, Phys. Rev. Lett. 106, 238103 (2011).

[42] É. Fodor, M. Guo, N. S. Gov, P. Visco, D. A. Weitz, and F. van Wijland, Activity-driven fluctuations in living cells, Europhys. Lett. 110, 48005 (2015).

[43] É. Fodor, W. W. Ahmed, M. Almonacid, M. Bussonnier, N. S. Gov, M.-H. Verlhac, T. Betz, P. Visco, and F. van Wijland, Nonequilibrium dissipation in living oocytes, Europhys. Lett. 116, 30008 (2016).

[44] E. Woillez, Y. Kafri, and V. Lecomte, Nonlocal stationary probability distributions and escape rates for an active Ornstein-Uhlenbeck particle, J. Stat. Mech. (2020) 063204.

[45] G. Szamel, Self-propelled particle in an external potential: Existence of an effective temperature, Phys. Rev. E 90, 012111 (2014).

[46] C. Maes, Response theory: A trajectory-based approach, Front. Phys. 8, 229 (2020).

[47] See Supplemental Material at http://link.aps.org/ supplemental/10.1103/PhysRevLett.125.208001 for more on linear response in the AOU medium and on the solution of the linear AOU model, which includes Refs. [48-51].

[48] L. Caprini, U. M. B. Marconi, and A. Vulpiani, Linear response and correlation of a self-propelled particle in the presence of external fields, J. Stat. Mech. (2018) 033203.

[49] Caleb G. Wagner, M. F. Hagan, and Aparna Baskaran, Response of active Brownian particles to boundary driving, Phys. Rev. E 100, 042610 (2019).

[50] M. Baiesi, C. Maes, and B. Wynants, Fluctuations and Response of Nonequilibrium States, Phys. Rev. Lett. 103, 010602 (2009).

[51] R. F. Fox, Functional-calculus approach to stochastic differential equations, Phys. Rev. A 33, 467 (1986).

[52] C. Maes, Frenesy: Time-symmetric dynamical activity in nonequilibria, Phys. Rep. 850, 1 (2020). 
[53] A. Dhar, A. Kundu, S. N. Majumdar, S. Sabhapandit, and G. Schehr, Run-and-tumble particle in one-dimensional confining potential: Steady state, relaxation and first passage properties, Phys. Rev. E 99, 032132 (2019).

[54] P. Baerts, U. Basu, C. Maes, and S. Safaverdi, The frenetic origin of negative differential response, Phys. Rev. E 88, 052109 (2013).

[55] A. Sarracino, F. Cecconi, A. Puglisi, and A. Vulpiani, Nonlinear Response of Inertial Tracers in Steady Laminar Flows: Differential and Absolute Negative Mobility, Phys. Rev. Lett. 117, 174501 (2016).

[56] U. Basu, P. de Buyl, C. Maes, and K. Netočný, Drivinginduced stability with long-range effects, Europhys. Lett. 115, 30007 (2016).
[57] C. Maes and T. Thiery, The induced motion of a probe coupled to a bath with random resettings, J. Phys. A 50, 415001 (2017).

[58] M. Krüger and C. Maes, The modified Langevin description for probes in a nonlinear medium, J. Phys. Condens. Matter 29, 064004 (2017).

[59] M. Baiesi, C. Maes, and B. Wynants, The modified Sutherland-Einstein relation for diffusive non-equilibria, Proc. R. Soc. A 467, 2792 (2011).

[60] T. Harada and S.-I. Sasa, Equality Connecting Energy Dissipation with a Violation of the Fluctuation-Response Relation, Phys. Rev. Lett. 95, 130602 (2005). 